\title{
Gerechtigkeit durch Fremdherrschaft? Zur moralischen Rechtfertigbarkeit von internationalen Übergangsverwaltungen
}

\author{
Daniel Jacob
}

Justice through Foreign Rule? On the Moral Justifiability of International Transitional Administrations

Abstract: Can international transitional administrations (ITAs) ever be morally justified, although they clearly constitute a form of foreign rule? The article addresses this question by relating it to the idea of a natural duty of justice. It thus can show that under certain conditions it is indeed morally justified to establish an ITA. At the same time, such an approach also highlights the limits of legitimate external interference, i.e. the distinction between morally required help and unjustifiable paternalism. As shown in the last part of the article, this distinction is of particular relevance for the task of evaluating the current practice of international transitional administrations.

Keywords: International transitional administrations, natural duty of justice, statebuilding, paternalism

Schlagwörter: Internationale Übergangsverwaltungen, natürliche Pflicht zur Gerechtigkeit, Staatszerfall, Statebuilding, Paternalismus

\section{Einleitung ${ }^{1}$}

Kann Fremdherrschaft jemals moralisch rechtfertigbar sein? Auf den ersten Blick scheint die Antwort eindeutig: Fremdherrschaft verletzt das Recht auf kollektive Selbstbestimmung, ist inhärent undemokratisch und somit moralisch nicht haltbar. Mit Lincolns Worten gesprochen handelt es sich weder um eine Regierung des Volkes noch um eine Regierung durch das Volk. Doch kann Fremdherrschaft möglicherweise trotzdem eine Regierung für das Volk darstellen? Seit Ende des Ersten Weltkrieges wurden internationale Übergangsverwaltungen eingerichtet, um zusammengebrochene staatliche Strukturen vorübergehend zu ersetzen und zugleich den Wiederaufbau ebendieser Strukturen zu ermöglichen. Jüngere Beispiele sind jene Übergangsverwaltungen, die ab Mitte der 1990er Jahre in Bosnien-Herzegowina, Kosovo und Ost-Timor eingerichtet wurden, sowie zuletzt die Übergangsverwaltung im Irak, die dort nach dem Krieg 2003 errichtet wurde. Jenseits einer Vielzahl praktischer Fragen wirft diese langjährige internationale Praxis die Frage auf, ob es nicht doch Umstände geben kann, unter denen bestimmte Formen von Fremdherrschaft moralisch rechtfertigbar sind.

1 Für hilfreiche Kommentare und Anregungen danke ich insbesondere Bernd Ladwig, Cord Schmelzle und Stefano Recchia sowie den beiden anonymen Gutachtern der „PVS“. Zudem danke ich der Deutschen Forschungsgesellschaft für die Förderung des Sonderforschungsbereichs 700 „Governance in Räumen begrenzter Staatlichkeit“, in dessen Kontext dieser Text entstanden ist. 
Um diese Frage zu beantworten, diskutiere ich internationale Übergangsverwaltungen in diesem Artikel aus einer gerechtigkeitstheoretischen Perspektive. Genauer frage ich, ob Übergangsverwaltungen als ein Mittel verstanden werden können, um die Anforderungen der natürlichen Pflicht zur Gerechtigkeit zu erfüllen. Dazu erläutere ich in einem ersten Schritt mein Verständnis internationaler Übergangsverwaltungen. In einem zweiten Schritt entwickele ich eine Konzeption der natürlichen Pflicht zur Gerechtigkeit, der als moralischer Kern eine Theorie menschlicher Grundinteressen zugrunde liegt. Vor diesem Hintergrund diskutiere ich im dritten Schritt, unter welchen Umständen die Einrichtung einer internationalen Übergangsverwaltung moralisch gerechtfertigt ist und worin die Ziele einer solchen Übergangsverwaltung bestehen sollten. Schließlich zeige ich anhand der internationalen Übergangsverwaltung im Kosovo, wie die hier angestellten Überlegungen als Maßstab zur moralischen Bewertung der bisherigen Praxis internationaler Übergangsverwaltungen dienen können.

Für Autoren wie William Bain (2003), David Chandler (2002) oder James Mayall (2011) stehen die in der gegenwärtigen Praxis vorgebrachten Begründungen für internationale Übergangsverwaltungen in der unrühmlichen Tradition liberaler Rechtfertigungen des Kolonialismus, wobei hier der moderne Menschenrechtsdiskurs die legitimatorische Funktion der „mission civilisatrice“ übernimmt. Folgt man diesen Kritikern, mag es fragwürdig erscheinen, die moralische Debatte über internationale Übergangsverwaltungen gerade aus Perspektive liberaler Gerechtigkeitstheorien führen zu wollen. Doch hoffe ich zeigen zu können, dass dieser Zugriff sehr wohl geeignet ist, das mit dem Vorwurf des Neo-Kolonialismus verbundene normative Anliegen zu erfassen. Dieses Anliegen, das von dem Selbstbestimmungsrecht der betroffenen Bevölkerung seinen Ausgangspunkt nimmt, lässt sich für die folgende Diskussion insbesondere in Form des gerade auch aus liberaler Perspektive zentralen Vorwurfs des Paternalismus reformulieren. Der Vorzug eines solchen Zugriffs besteht dann darin, die aus liberaler Perspektive erwachsenden Gründen für internationale Übergangsverwaltungen systematisch mit diesen Einwänden im Lichte der kolonialen Geschichte verbinden zu können.

\section{Internationale Übergangsverwaltungen}

In der empirischen Forschung zu internationalen Übergangsverwaltungen werden eine Reihe von sich zum Großteil überlappenden Begrifflichkeiten verwendet. So benutzt Simon Chesterman (2004, S. 5) den Begriff der „transitional administration “, um eine besonders umfassende Form von Statebuilding zu beschreiben, bei der externe Akteure vorübergehend die Befugnisse der lokalen Regierung in einigen oder gar allen relevanten Hinsichten für sich in Anspruch nehmen. Für Richard Caplan (2005, S. 2) sind „international administrations“ hingegen gerade dadurch gekennzeichnet, dass sie in Umfang und Anspruch über klassische Formen von Statebuilding hinausgehen. Ralph Wilde (2001, S. 585) schlägt den Begriff der „international territorial administration“ vor und versteht darunter eine formal konstituierte, lokal operierende und auf ein klar abgegrenztes Territorium bezogene Verwaltungsstruktur. Bothe und Marauhn (2002, S. 219) diskutieren Kosovo und Ost-Timor als Fälle von „trusteeship administration“ und betonen 
so das treuhänderische Mandat von Übergangsverwaltung. Und in einem kurzen Artikel zur Verantwortung der internationalen Gemeinschaft für den Wiederaufbau des Irak spricht Michael Walzer schlicht (2004) von „occupation“, in ähnlicher Weise verwendet Gregory Fox (2008) den Begriff der „humanitarian occupation ". All diese Begrifflichkeiten und die zugrundeliegenden Definitionen betonen unterschiedliche Aspekte dessen, was internationale Übergangsverwaltungen auszeichnet. Es scheint daher sinnvoll, nicht einfach eine dieser Begrifflichkeiten zu übernehmen, sondern diese vielmehr zusammenzuführen. Im Folgenden verstehe ich demzufolge internationale Übergangsverwaltungen als eine besondere Form von Fremdherrschaft, welche (1) auf einer militärischen Besatzung berubt, (2) einen Anspruch auf das Gewaltmonopol und die Autorität zur Setzung und Implementierung kollektiv verbindlicher Entscheidungen erhebt und (3) zeitlich beschränkt ist und zum Ziel hat, den Aufbau lokaler Regierungsinstitutionen zu unterstützen.

Im Anschluss an Walzer und Fox betont das erste Element dieser Definition, dass internationale Übergangsverwaltungen sich als Form militärischer Besatzung auf die umfangreiche Stationierung externer militärischer Truppen stützen. Dies schließt andere Formen nicht-militärischer Einflussnahme externer Akteure auf lokale politische Prozesse aus, wenngleich diese unter Umständen ähnliche Fragen aufwerfen. ${ }^{2}$ Die Knüpfung von Entwicklungshilfe an politische Bedingungen etwa kann durchaus bedeuten, dass externe Akteure wie die Weltbank erheblichen Einfluss auf die politischen Entwicklungen in einem Land ausüben können (Grant 2012, S. 101-111). Doch auch wenn man diese Form der Einflussnahme in gewisser Verwendung des Begriffs auch als eine Form von Fremdherrschaft beschreiben kann, ist sie nicht mit militärischer Besatzung gleichzusetzen.

Gemäß dem zweiten Element der hier vorgeschlagenen Definition erheben internationale Übergangsverwaltungen den Anspruch auf das Gewaltmonopol und die Autorität zur Setzung und Umsetzung kollektiv bindender Entscheidungen. Auch wenn darüber hinaus der Umfang der Aktivitäten von Übergangsverwaltungen variiert (Chesterman 2004, S. 5; Wilde 2001, S. 585), markiert dies einen zentralen Unterschied zu anderen Formen des Statebuilding. Auch wird so deutlich, warum das Handeln externer Akteure in Afghanistan nach 2001 keinen Fall einer internationalen Übergangsverwaltung darstellt. Zwar stellen sich hier ohne Zweifel ähnliche Fragen wie im Falle internationaler Übergangsverwaltungen. Und doch unterscheidet sich Afghanistan dadurch, dass unmittelbar nach Ende der Kriegshandlungen eine afghanische Regierung gebildet wurde, die seitdem auch gegenüber den externen Akteuren wenigstens formal Anspruch auf das Gewaltmonopol und die Autorität zur Setzung und Implementierung kollektiv bindender Entscheidungen erhebt (Suhrke 2009, S. 230-243).

Das dritte Element der hier vorgeschlagenen Definition schließlich betont den temporären Charakter von internationalen Übergangsverwaltungen und schreibt diesen einen Zweck zu, der sie von anderen Formen der Fremdherrschaft unter-

$2 \mathrm{Ob}$ internationale Übergangsverwaltungen auch als Besatzungen im engeren völkerrechtlichen Sinne gelten sollten, ist jedoch umstritten. Siehe für diese Debatte Sassòli (2005, S. 686-693) und Fox (2008, Kapitel 7). 
scheidet. War Fremdherrschaft traditionell auf die langfristige Ausweitung des eigenen Machtbereichs angelegt, so besteht der Zweck von Übergangsverwaltungen vielmehr darin, den Aufbau lokaler Regierungsinstitutionen zu unterstützen. Wie Robert Jackson (2004, S. 26) formuliert, ist eine Übergangsverwaltung „in business to put itself out of business".

Historisch betrachtet sind internationale Übergangsverwaltungen bereits seit fast hundert Jahren ein zwar seltenes, aber doch regelmäßig wiederkehrendes Instrument internationaler Politik. Die durch den Völkerbund mandatierte Verwaltung des Saarlandes durch Frankreich und Großbritannien von 1919 bis 1935 war der historisch erste Fall einer solchen Übergangsverwaltung. Die prominentesten historischen Beispiele sind jedoch die Verwaltung Deutschlands, Österreichs und Japans durch die Alliierten nach dem Ende des Zweiten Weltkrieges. Während des Kalten Krieges gab es nur einen einzigen Fall einer internationalen Übergangsverwaltung, die von 1962 bis 1963 West Papua verwaltete und unter kontroversen Umständen die Eingliederung in den indonesischen Staat durchführte.

Die erste internationale Übergangsverwaltung nach dem Ende des Kalten Krieges wurde 1997 in Bosnien-Herzegowina eingerichtet. Obschon der BosnienKrieg bereits 1995 durch das Abkommen von Dayton beendet wurde, fürchteten die im Friedensprozess involvierten Staaten einen neuen Gewaltausbruch und entschieden sich so zur Einrichtung einer internationalen Übergangsverwaltung, die bis heute fortbesteht. Ebenfalls in Folge des Bosnien-Krieges wurde eine weitere internationale Übergangsverwaltung in Ost-Slawonien eingerichtet, deren Funktion vor allem darin bestand, die Eingliederung dieses Gebietes in den kroatischen Staat zu organisieren. Nach der militärischen Intervention der NATO im Kosovo wurde 1999 schließlich eine weitere internationale Übergangsverwaltung auf dem Gebiet des ehemaligen Jugoslawiens errichtet, die dann 2008 mit der Unabhängigkeitserklärung des Kosovos beendet wurde. Ebenfalls 1999 wurde in Ost-Timor eine UN-geführte internationale Übergangsverwaltung eingerichtet. Nach gewaltsamen Auseinandersetzungen um die Unabhängigkeit Ost-Timors waren große Teile der Infrastruktur des Landes zerstört worden. Bis zur offiziellen Staatsgründung 2002 war es daher Aufgabe der Übergangsverwaltung, neben unmittelbaren Hilfsleistungen den Aufbau staatlicher Institutionen zu unterstützen. Der jüngste Fall einer internationalen Übergangsverwaltung war die „Coalition Provisional Authority “, die von 2003 bis 2004 unter Führung der USA den Wiederaufbau im Irak organisieren sollte. ${ }^{3}$

3 Zum Saarland siehe Stahn (2008a, S. 163-173) und Wambaugh (1940, Kapitel 1). Zu Deutschland siehe Diefendorf et al. (1993) und Jarausch (2006). Zu Österreich siehe Bader (1966) und Carafano (2002). Zu Japan siehe Dower (1999) und Kawai (1979). Zu West Papua siehe Gruss (2005) und Saltford (2003). Zu Bosnien-Herzegowina siehe Bose (2002) und Caplan (2004a). Zu Ostslawonien siehe Boothby (2004) und Caplan (2004b, S. 232-233). Zu Kosovo siehe King u. Mason (2006) und Weller (2008). Zu Ost-Timor siehe Chesterman (2002) und Wheeler u. Dunne (2001). Zu Irak siehe Dawisha (2009) und Wolfrum (2005). 


\section{Die natürliche Pflicht zur Gerechtigkeit}

Die Idee einer natürlichen Pflicht zur Gerechtigkeit wurde zuerst von John Rawls in seinen Überlegungen zur Theorie der Gerechtigkeit (1975) eingeführt. Dort beschreibt er sie als die Pflicht, „vorhandene und für uns geltende gerechte Institutionen zu unterstützen und ihre Regeln zu beachten. Sie verlangt von uns auch die Förderung noch nicht verwirklichter gerechter Regelungen, zumindest wenn uns das ohne allzu große Nachteile möglich ist" (Rawls 1975, S. 137). Die natürliche Pflicht zur Gerechtigkeit ergänzt so auf der Ebene des Individuums Rawls' Prinzipien für gerechte Institutionen. Dabei versteht Rawls vor dem Hintergrund seiner konstruktivistischen Gerechtigkeitstheorie das Attribut „natürlich“ nicht als essentialistische Aussage über die moralische Natur des Menschen. Vielmehr will er mit diesem Attribut natürliche Pflichten, die uns allen in gleicher Weise zukommen, von jenen spezifischeren Verpflichtungen unterscheiden, die wir erst durch eigenes Handeln oder besondere Beziehungen zu anderen Menschen eingehen (Rawls 1975, S. 135-136).

Allen Buchanan hat diesen Vorschlag jüngst in einer Weise aktualisiert, die eine direkte Verbindung zur Idee der Menschenrechte herstellt. So beschreibt Buchanan die natürliche Pflicht zur Gerechtigkeit als „the limited moral obligation to contribute to ensuring that all persons have access to just institutions, where this means primarily institutions that protect basic human rights" (2004, S. 86). Im Folgenden nehme ich diese Überlegungen zur natürlichen Pflicht zur Gerechtigkeit zum Ausgangspunkt. Ich schlage jedoch vor, sie in dreierlei Hinsichten zu erweitern: Erstens durch eine Konzeption menschlicher Grundinteressen; zweitens durch ein mit der Idee der Menschenrechte kompatibles Verständnis des Rechts auf kollektive Selbstbestimmung; drittens durch die Idee einer globalen moralischen Arbeitsteilung. Durch diese Erweiterungen wird es möglich, genauer zu bestimmen, welche Art von öffentlichen Institutionen wir im Sinne der natürlichen Pflicht zur Gerechtigkeit unterstützen bzw., falls noch nicht vorhanden, aufbauen sollten.

\subsection{Menschliche Grundinteressen}

Die natürliche Pflicht zur Gerechtigkeit verweist nach Buchanan im Kern auf die Idee der Menschenrechte. Wie er betont, sind dabei moralisch jedoch letztlich nicht diese Rechte ausschlaggebend, sondern jene Interessen, die durch diese Rechte geschützt werden (Buchanan 2004, S. 124-127). Obwohl Buchanan damit für eine Interessenkonzeption der Menschenrechte argumentiert, deutet er nur an einigen Stellen an, welche Interessen er in diesem Zusammenhang für moralisch relevant hält. An dieser Stelle bietet es sich an, Buchanans Verständnis von Menschenrechten durch eine explizit normative Konzeption menschlicher Grundinteressen zu ergänzen. Die Idee menschlicher Grundinteressen ist dabei als Erläuterung der Annahme zu verstehen, dass allen Menschen ein gleicher moralischer Status zukommt (Anderson 1999, S. 312). Sie bildet den Versuch, über den Weg solcher Interessen genauer zu bestimmen, worin dieser gleiche moralische Status besteht. So verstanden eignet sich die Idee menschlicher Grundinteressen in be- 
sonderer Weise, um den Inhalt der natürlichen Pflicht zur Gerechtigkeit zu bestimmen: Als moralisch Gleichen kommt uns demnach die reziproke Pflicht zu, zum institutionellen Schutz dieser Grundinteressen beizutragen. ${ }^{4}$

Doch wie ist es möglich, Interessen zu benennen, die moralisch relevant, hinreichend substanziell und dennoch verallgemeinerbar sind? Im Anschluss an Bernd Ladwig (2010, S. 64-65) und David Miller (2007, S. 185-194) ist mein Vorschlag hier, dass Interessen zwei Kriterien erfüllen müssen, um als menschliche Grundinteressen gelten zu können: Wenn die Idee menschlicher Grundinteressen uns dabei helfen soll, den Inhalt der natürlichen Pflicht zur Gerechtigkeit zu bestimmen, muss es erstens möglich sein, menschliche Grundinteressen in hierzu korrespondierende Pflichten zu übersetzen. Dies schließt etwa Interessen aus, die aufgrund der Natur des Interesses nur durch freiwillige Handlungen Anderer erfüllt werden können. Auch wenn wohl die meisten Menschen ein hohes Interesse daran haben, geliebt zu werden, lässt sich dieses Interesse nicht in eine korrespondierende Pflicht übersetzen. Sollen menschliche Grundinteressen zweitens auch unter den Bedingungen des ethischen Pluralismus verallgemeinerbar sein, müssen sie auf jene Interessen beschränkt sein, deren Schutz für ein minimal gutes Leben notwendig ist. Dieser Fokus auf die Bedingungen eines minimal guten Lebens markiert einen wichtigen Unterschied zu perfektionistischen Theorien des Guten und macht die Idee menschlicher Grundinteressen kompatibel zu einer Vielzahl von Konzeptionen des Guten, ohne sich dabei jedoch in völliger Neutralität zu verlieren (Arneson 1980; Nussbaum 2011). Legt man diese beiden Kriterien zugrunde, so lassen sich zwei menschliche Grundinteressen identifizieren: das Interesse an körperlicher Unversehrtheit und das Interesse an Autonomie.

Das Interesse an körperlicher Unversehrtheit ist zu verstehen als das Interesse an der Vermeidung von nicht-trivialen, vermeidbaren Formen körperlichen Leidens. Es hat zwei Dimensionen, die beide korrespondierende Pflichten begründen: nicht von anderen verletzt zu werden und Zugang zu ausreichenden Subsistenzmitteln zu haben. Die Bedeutung des Schutzes körperlicher Unversehrtheit für die Möglichkeit, ein minimal gutes Leben zu führen, kann kaum bestritten werden. Körperliche Unversehrtheit im hier beschriebenen Sinne stellt in existenzieller Weise die Voraussetzung dafür dar, eine Konzeption des Guten entwickeln und verfolgen zu können. Doch ist körperliche Unversehrtheit nicht nur instrumentell bedeutsam für die Möglichkeit, ein gutes Leben zu führen, sondern selbst Teil dessen, was ein minimal gutes Leben ausmacht (Ladwig 2010, S. 63-64).

Das menschliche Grundinteresse an Autonomie geht über die physische Dimension menschlicher Existenz hinaus und formuliert das Interesse daran, eigenständig Wünsche und Überzeugungen ausbilden und im Einklang mit diesen handeln zu können. ${ }^{5}$ Diesem Interesse an einer autonomen Lebensführung liegt dabei ein Verständnis von Autonomie als Fähigkeit zugrunde. Diese Fähigkeit kann unter-

4 Ein solches Verständnis von menschlichen Grundinteressen kommt Feinbergs Idee von „welfare interests“ (1984, S. 37) recht nahe, ebenso wie Höffes Überlegungen zu „transzendentalen Interessen“" (1998).

5 Für einen Überblick zur jüngeren Debatte um den Autonomiebegriff siehe Anderson u. Christman (2005) und Mackenzie u. Stoljar (2000). 
schiedlich stark ausgeprägt sein, setzt jedoch in jedem Fall ein Mindestmaß an Reflexivität voraus, das darin besteht, die eigenen Überzeugungen und Handlungen in Frage stellen und ggf. verändern zu können (Feinberg 1986, S. 30). ${ }^{6}$ Die dem menschlichen Grundinteresse an Autonomie korrespondierende Pflicht orientiert sich an diesem Mindestmaß an Reflexivität: Sie verlangt, die Autonomie anderer wenigstens soweit zu achten und zu schützen, dass ein solches Maß an reflexiver Lebensführung möglich ist.

Für die folgende Diskussion bedeutsam ist dabei, dass das menschliche Grundinteresse an Autonomie auch eine kollektive Dimension hat. Zum einen, wie insbesondere von kommunitaristischen und feministischen Autoren betont wird, ist individuelle Autonomie immer in einen sozialen Kontext eingebettet, der Autonomie erst ermöglicht, bisweilen aber auch beschränkt (Christman 2009; Mackenzie u. Stoljar 2000, S. 5-12). Zum anderen kann die Ausübung von Autonomie kollektive Formen annehmen, dann nämlich wenn wir für die Verfolgung unserer Ziele auf die Kooperation mit anderen angewiesen sind. Wenn wir eine Familie gründen, im Chor singen oder eine politische Partei bilden, so üben wir in diesem Sinne kollektive Autonomie aus (Pettit u. Schweikard 2006).

So verstanden ist Autonomie von zentraler Bedeutung für die Möglichkeit, ein minimal gutes Leben zu leben. Um überhaupt irgendeine Vorstellung vom guten Leben entwickeln und dementsprechend handeln zu können, müssen wir die Möglichkeit haben, wenigstens in dem hier beschriebenen minimalen Sinne von unserer Fähigkeit zur Autonomie Gebrauch machen zu können. Ein naheliegender Einwand an dieser Stelle lautet, dass nicht alle Menschen in gleicher Weise ein Interesse an einer autonomen Lebensführung haben. Dieser Einwand verfehlt jedoch den moralischen Stellenwert der Idee eines menschlichen Grundinteresses an Autonomie. Ein solches Grundinteresse impliziert nicht, dass nur ein in jeglicher Hinsicht autonomes Leben ein gutes Leben sein kann, sollte so etwas überhaupt je möglich sein. Entscheide ich mich, langfristige soziale Bindungen einzugehen - etwa zu heiraten oder mich den Regeln einer religiösen Gemeinschaft zu unterwerfen - so schränkt dies in gewissen Hinsichten meiner täglichen Lebensführung meine Autonomie ein. Solange diese Selbstbeschränkung auf einer höherstufigen Ebene Ergebnis meiner eigenen Entscheidung ist und von mir widerrufen werden kann, lässt sich dies jedoch selbst als zentrales Element meiner Autonomie verstehen. Moralisch unzulässig wäre es hingegen, wenn mir von anderen eine derartige Beschränkung meiner Autonomie aufgezwungen würde (Nussbaum 2000, S. 92-96).

\subsection{Die institutionellen Anforderungen der natürlichen Pflicht zur Gerechtigkeit}

Im Anschluss an Buchanan verstehe ich Menschenrechte als ein Instrument zum Schutz menschlicher Grundinteressen. Wie auch Buchanan bemerkt, sind diese Interessen für sich genommen jedoch zu abstrakt, um hinreichend normative Orientierung zu bieten. Demgegenüber schafft die Übersetzung in Rechte gleich in zweierlei Hinsicht Klarheit: Sie formuliert in größerer Genauigkeit, was wir ein-

6 Siehe hierzu auch Christman (2005, S. 333-335), Dworkin (1989, S. 61), Frankfurt (1971) und Raz (1986, S. 372-373). 
ander in unseren direkten Interaktionen schulden und sie liefert vor allem Maßstäbe dafür, welche Arten von Institutionen wir unterstützen sollten. So verstanden verlangt die natürliche Pflicht zur Gerechtigkeit von uns, jene Institutionen zu unterstützen, die zu dem Schutz dieser Rechte beitragen.

Ohne dies hier ausführlich darstellen zu können umfassen die Menschenrechte zum einen solche Rechte, die das menschliche Grundinteresse auf körperliche Unversehrtheit schützen: Hierzu zählen mit dem Recht auf Leben und dem Recht auf Schutz vor Folter jene Rechte, die James Nickel (2007, S. 92-95) als „Sicherheitsrechte" beschreibt, aber auch das Recht auf Zugang zu grundlegenden Subsistenzmitteln (Shue 1980, S. 23). Zum anderen schützen die traditionellen Freiheitsrechte sowie grundlegende politische und soziale Rechte das menschliche Grundinteresse an einer autonomen Lebensführung - und sind demnach auch den Menschenrechten zuzuschreiben. So sind grundlegende politische Rechte notwendig, um jenseits der Privatsphäre autonomes Handeln zu ermöglichen. In diesem Sinne sichert etwa ein so grundlegendes soziales Recht wie das Recht auf Bildung die Möglichkeit, die Fähigkeit zu autonomem Denken und Handeln überhaupt erst ausbilden zu können. Ein zentraler Einwand gegen ein solch vergleichsweise umfassendes Verständnis von Menschenrechten lautet, dass dieses in unzulässiger Weise das Recht auf kollektive Selbstbestimmung begrenze. ${ }^{7}$ Diesem Argument lässt sich jedoch begegnen, wenn wir auch das Recht auf kollektive Selbstbestimmung als Instrument zum Schutz menschlicher Grundinteressen verstehen.

Das Recht auf kollektive Selbstbestimmung spricht einer politischen Gemeinschaft das Recht zu, über ihre internen Angelegenheiten ohne Einmischung von außen entscheiden zu dürfen. Es schützt bezogen auf den Bereich des Politischen das menschliche Grundinteresse an Autonomie in seiner kollektiven Dimension, und verhält sich so komplementär zu den Menschenrechten: Freiheitsrechte sowie grundlegende politische Rechte schützen die Autonomie der einzelnen Mitglieder einer politischen Gemeinschaft mit Blick auf deren interne Beziehungen; das Recht auf kollektive Selbstbestimmung erweitert diesen Schutz mit Blick auf die externen Beziehungen einer politischen Gemeinschaft.

Zusammen liefern die Menschenrechte und das Recht auf kollektive Selbstbestimmung so schließlich die Kriterien dafür, welche Funktionen öffentliche Institutionen erfüllen müssen, um als wenigstens minimal gerecht zu gelten. Die natürliche Pflicht zur Gerechtigkeit verlangt demnach von uns, Institutionen zu unterstützen oder - wo sie fehlen - aufzubauen, die in diesem Sinne minimal gerecht sind.

Ein Staat kann als eine solche minimal gerechte Institutionen verstanden werden, wenn drei Bedingungen erfüllt sind: Erstens muss ein Staat willens und in der Lage sein, die Menschenrechte seiner Bürger und ihr Recht auf kollektive Selbstbestimmung zu schützen. Zweitens muss ein Staat sich selbst als Teil einer globalen moralischen Arbeitsteilung verstehen. Es erscheint auf den ersten Blick widersprüchlich, die im Kern kosmopolitische, weil eben natürliche, Pflicht zur Gerechtigkeit mit immer partikularen staatlichen Institutionen zu verknüpfen.

7 Für eine Übersicht zu den Kontroversen über die Idee von politischen und sozialen Menschenrechten siehe Beitz (2009) und Nickel (2007). 
Dieser vermeintliche Widerspruch lässt sich jedoch auflösen, wenn man Staaten als Teil einer globalen moralischen Arbeitsteilung versteht (Shue 1988; Goodin 1988). Im Rahmen dieser Arbeitsteilung kommt Staaten eine primäre Verantwortung gegenüber ihren Bürgern zu. Darüber hinaus kommt ihnen als Mitglied der Staatengemeinschaft zudem eine subsidiäre Verantwortung gegenüber der gesamten Menschheit zu. Diese subsidiäre Verantwortung verlangt von der Staatengemeinschaft, (a) sicherzustellen, dass Staaten ihrer primären Verantwortung gerecht werden und (b) Hilfe zu leisten, wo ein Staat dies nicht tut. Drittens gilt diese Rechtfertigung von Staaten und dem Staatensystem in Gänze nur solange, wie es keine plausible institutionelle Alternative gibt. Ohne die Schattenseiten des Staatensystems in Abrede zu stellen, scheint dies derzeit noch der Fall zu sein (Waldron 1993, S. 20-25; Wellman 1996, S. 219; Stilz 2009, S. 47-52). Insofern als Staaten aus der Perspektive der natürlichen Pflicht zur Gerechtigkeit primär eine instrumentelle Bedeutung zukommt, würde ihr moralischer Status jedoch in dem Moment in Frage gestellt werden, in dem andere Instrumente, etwa eine weiterentwickelte Form der Europäischen Union, sich zu realistischen institutionellen Alternativen entwickeln.

\section{Gerechtigkeit durch Fremdherrschaft?}

Im Lichte der bisherigen Erläuterungen zur natürlichen Pflicht zur Gerechtigkeit lässt sich nun fragen, ob internationale Übergangsverwaltungen als ein geeignetes Mittel verstanden werden können, um die Anforderungen dieser Pflicht zu erfüllen. Genauer stellt sich die Frage, ob solche Übergangsverwaltungen ein Mittel sein können, durch das die Staatengemeinschaft ihrer subsidiären Verantwortung in jenen Fällen gerecht werden kann, in denen einzelne Staaten ihrer primären Verantwortung gegenüber ihren Bürgern nicht nachkommen. Im Gegensatz zur bisherigen Diskussion der natürlichen Pflicht zur Gerechtigkeit verschiebt sich damit der Fokus. Wurde die natürliche Pflicht zur Gerechtigkeit bisher als individuelle Pflicht diskutiert, so geht es nun darum, inwiefern Staaten sich in ihrem Handeln an den Anforderungen dieser Pflicht orientieren sollten. Diese Verschiebung ist jedoch nur pragmatischer Natur und in gewisser Weise metaphorisch: Die Pflichten eines Staates verweisen letztlich wiederum auf die Pflichten der Repräsentanten und Bürger dieser Staaten, von ihren politischen Handlungsmöglichkeiten auf eine bestimmte Weise Gebrauch zu machen (Pogge 2005, S. 78-83).

\subsection{Internationale Übergangsverwaltungen als Antwort auf extremen Staatszerfall}

Die Idee einer globalen moralischen Arbeitsteilung bringt ein normatives Ideal zum Ausdruck, das die Schwächen des gegenwärtigen Staatensystems deutlich hervortreten lässt. Viele Staaten sind nicht in der Lage oder nicht willens, die Menschenrechte ihrer Bürger und ihr Recht auf kollektive Selbstbestimmung zu schützen. Gleichzeitig ist auch das Staatensystem in Gänze, derzeit vor allem durch die Vereinten Nationen verkörpert, weit davon entfernt, seiner subsidiären Verantwortung gerecht zu werden. Die Diskussion um die „responsibility to pro- 
tect" kann als Versuch verstanden werden, hier Abhilfe zu schaffen. Im Zentrum dieser Diskussion stand zunächst die Frage, wie mit Staaten umzugehen ist, die selbst aktiv die Rechte ihrer Bürger verletzen. Insbesondere wurde im Anschluss an die Tradition der Theorie des Gerechten Krieges erörtert, ob in solchen Fällen militärische Interventionen gerechtfertigt sein können (Holzgrefe u. Keohane 2003). Die Diskussion der moralischen Rechtfertigbarkeit von internationalen Übergangsverwaltungen hingegen knüpft an einen bisher weitgehend vernachlässigten Aspekt der Debatte um die „responsibility to protect“ an, nämlich die Frage, ob diese auch eine „responsibility to rebuild“ beinhalte. ${ }^{8}$ Im Mittelpunkt stehen hier vor dem Hintergrund der Zunahme des Phänomens zerfallener Staatlichkeit solche Staaten, die die Rechte ihrer Bürger schlicht nicht schützen können, selbst wenn sie wollten (Patrick 2006, S. 27-53; Call 2010; Rotberg 2004). Derartige Situationen werfen praktisch wie moralisch Fragen auf, die über die Debatte zu „humanitären Interventionen“ hinausweisen.

Nicht kontrovers erscheint dabei zunächst, dass die subsidiäre Verantwortung der Staatengemeinschaft auch die Pflicht beinhaltet, Staaten zu helfen, die ihrer primären Verantwortung nicht nachkommen können. Ziel dieser Hilfe muss es sein, durch eine Kombination von kurzfristigen Hilfsmaßnahmen und längerfristig angelegter Entwicklungszusammenarbeit den betreffenden Staat in die Lage zu versetzen, die Rechte seiner Bürger zuverlässig schützen zu können. Unter Umständen kann dies auch Reformen des globalen politischen und ökonomischen Systems notwendig machen, dann nämlich, wenn dieses System die Ursache für den Zusammenbruch eines Staates darstellt (Pogge 2004).

In Fällen von extremem Staatszerfall jedoch reicht dies nicht aus. Extremer Staatszerfall unterscheidet sich von anderen Formen zerfallener Staatlichkeit durch die Unfähigkeit des Staates, jene grundlegenden Sicherheitsrechte seiner Bürger institutionell abzusichern, die in existenzieller Weise das menschliche Grundinteresse an körperlicher Unversehrtheit schützen: das Recht auf Leben und das Recht auf Schutz vor Folter. Ist ein Staat nicht mehr in der Lage, diese Rechte zu schützen, so bekommt die Hilfe durch externe Akteure eine besondere Dringlichkeit. Während im Falle des unzureichenden Schutzes anderer Menschenrechte mittel- bis langfristige Entwicklungszusammenarbeit zur Stärkung lokaler Regierungsinstitutionen ein angemessenes Mittel sein kann, um den Bürgern des betroffenen Staates zu helfen, so besteht diese Option hier nicht. Die Bürger extrem zerfallener Staaten sehen sich existenziellen Bedrohungen ausgesetzt und können nicht auf die Effekte mittel- bis langfristiger Maßnahmen warten. Neben dieser besonderen moralischen Dringlichkeit ist es dabei der besondere Typ von Bedrohung, der in solchen Fällen die Einrichtung einer internationalen Übergangsverwaltung rechtfertigt und letztlich sogar als moralisch geboten erscheinen lässt. Wie oben erläutert, sind solche Übergangsverwaltungen unter anderem darüber definiert, dass sie den Anspruch auf das Gewaltmonopol erheben und über die militärischen und polizeilichen Ressourcen verfügen, um diesen Anspruch auch effektiv durchzusetzen. Eben dies ist notwendig, um in Situationen von extremem

8 Für einen Überblick zur moralischen Debatte um die „responsibility to protect“ siehe Bellamy (2010). Zur „responsibility to rebuild“ siehe Gheciu u. Welsh (2009). 
Staatszerfall einen effektiven Schutz der Sicherheitsrechte der betroffenen Bevölkerung zu gewährleisten.

Versteht man eine internationale Übergangsverwaltung in diesem Sinne als Reaktion auf extremen Staatszerfall, so ergibt sich hieraus bereits eine erste zentrale Aufgabe einer solchen Übergangsverwaltung: eben der Schutz jener Sicherheitsrechte, die sich aus dem menschlichen Grundinteresse an körperlicher Unversehrtheit ergeben. Um dieser Aufgabe nachzukommen, kann sich eine Übergangsverwaltung verbliebener lokaler Strukturen bedienen. Solange der betroffene Staat selbst noch nicht wieder in der Lage ist, die Sicherheitsrechte seiner Bürger zuverlässig zu schützen, liegt die Letztverantwortung hierfür jedoch bei der Übergangsverwaltung.

Neben der Letztverantwortung für den Schutz grundlegender Menschenrechte muss eine internationale Übergangsverwaltung zudem den Wiederaufbau minimal gerechter Institutionen sicherstellen. Die zentrale Rolle muss hierbei zweifelsohne soweit wie möglich lokalen Akteuren zukommen. Die Konkretisierung der oben diskutierten Maßstäbe für minimal gerechte Institutionen ist schließlich selbst ein zentrales Element des Rechts auf kollektive Selbstbestimmung. Dementsprechend sollte die Rolle einer internationalen Übergangsverwaltung hier vorwiegend unterstützender Natur sein. Darüber hinaus muss eine Übergangsverwaltung aber auch sicherstellen, dass die Wiederaufbaumaßnahmen nicht in Widerspruch zum Ziel minimal gerechter Institutionen geraten. Ein solcher Widerspruch kann etwa durch den Aufbau eines Schulwesens geschehen, das Mädchen den Zugang zu Bildung verwehrt und damit direkt das menschliche Grundinteresse an Autonomie verletzt.

Dabei ist zu betonen, dass dieses Ziel Raum für eine Vielzahl von Ausprägungen staatlicher Institutionen lässt. Minimal gerechte Institutionen müssen nicht dem stereotypen Ideal des skandinavischen Wohlfahrtsstaates entsprechen. Sie müssen auch nicht ausschließlich aus rein staatlichen Institutionen bestehen, sondern können auch nicht-staatlichen Akteuren bestimmte Governance-Funktionen zuweisen (Börzel u. Risse 2010). Entscheidend ist lediglich, dass minimal gerechte Institutionen zuverlässig die Menschenrechte der Bevölkerung und ihr Recht auf kollektive Selbstbestimmung schützen. Ist dieses immer noch anspruchsvolle, aber explizit nicht-perfektionistische, Ziel erreicht, muss eine internationale Übergangsverwaltung beendet werden. Zwar kann auch jenseits dieses Zieles weitere Entwicklungszusammenarbeit geboten sein, diese muss dann jedoch die Form einer genuinen Zusammenarbeit zwischen externen Akteuren und der lokalen Regierung annehmen.

\subsection{Die Gefahr des Paternalismus}

Der aus moralischer Perspektive zentrale Einwand gegen internationale Übergangsverwaltungen lautet, dass diese in paternalistischer Weise das Recht der betroffenen Bevölkerung auf kollektive Selbstbestimmung verletzten. Wie bereits in der Einleitung angedeutet, lässt sich dies auch als eines der zentralen Anliegen jener Autoren verstehen, die internationale Übergangsverwaltungen als neo-kolonialistische Unternehmungen kritisieren. 
Gerald Dworkin definiert Paternalismus als „the interference with a person's liberty of action justified by reasons referring exclusively to the welfare, good, happiness, needs, interests, or values of the person being coerced" (Dworkin 1983, S. 20). Paternalismus hat demnach zwei Komponenten: eine Handlung, welche die Freiheit einer anderen Person - oder auch einer Gruppe (Neuhäuser 2011) - einschränkt und eine besondere Art der Rechtfertigung für diese Handlung. Dabei ist es gerade die Kombination dieser zwei Komponenten, die paternalistische Handlungen von anderen Handlungen unterscheidet. So ist es schließlich möglich, die Freiheit anderer aus weit weniger wohlwollenden Gründen zu beschränken, und umgekehrt ist es ebenso möglich, das Wohl einer anderen Person auf andere Weise zu fördern (Grill 2007; Feinberg 1986, S. 4). Handelt Person A nun paternalistisch gegenüber Person B, so ist dies in besonderer Weise moralisch problematisch, nicht nur weil Bs Freiheit eingeschränkt wird, sondern vor allem weil A dabei B, zumindest in den für die Handlung relevanten Hinsichten, die Fähigkeit zu Autonomie abspricht. A rechtfertigt die Freiheitsbeschränkung von B schließlich damit, dass B selbst nicht in der Lage sei, zu erkennen, was für ihn gut sei, und entsprechend zu handeln. Ob es dennoch Umstände geben kann, unter denen Paternalismus auch aus liberaler Perspektive rechtfertigbar sein kann, bleibt umstritten (Dworkin 2010). So argumentiert etwa Peter de Marneffe (2005), dass bestimmte Formen des Paternalismus - wie etwa die staatliche Helmpflicht für Motorradfahrer - durchaus mit der liberalen Betonung von Autonomie vereinbar seien. Dabei bezweifelt jedoch auch Marneffe nicht, dass die paternalistische Einschränkung grundlegender Freiheitsrechte ein moralisches Übel und eine besondere Form der Demütigung darstellt.

Angewandt auf den Fall internationaler Übergangsverwaltungen lautet eine erste Variante des Paternalismus-Einwandes, dass derartige Übergangsverwaltungen die Autonomie der lokalen Bevölkerung in unzulässiger Weise einschränken, wenn sie an die Stelle von lokalen Regierungsinstitutionen treten. Dieser Einwand lässt sich jedoch entkräften, wenn man sich bewusst macht, dass gerade kollektive Autonomie an bestimmte Voraussetzungen gebunden ist. In einer Situation, in der die öffentlichen Institutionen eines Staates nahezu vollständig zusammengebrochen sind und die Bürger dieses Staates permanent um ihr Leben fürchten müssen, sind diese Voraussetzungen auf ganz grundlegende Weise nicht erfüllt. Zwar werden viele Bürger eines solchen Staates in vielen Hinsichten weiterhin in der Lage sein, auf der individuellen Ebene ihre Autonomie zu bewahren, doch ist es den Bürgern eines solchen Staates nicht möglich, als politische Gemeinschaft kollektive Entscheidungen zu treffen, geschweige denn umzusetzen. So verfügen extrem zerfallene Staaten in aller Regel nicht über die Institutionen, die für gemeinsame Entscheidungsfindung sowie für die Umsetzung der gemeinsam getroffenen Entscheidungen notwendig sind. Vor allem aber sind sie eben nicht in der Lage, die sichere Teilnahme an kollektiven Entscheidungsfindungsverfahren zu gewährleisten. In einer solchen Situation bedeutet die Errichtung einer internationalen Übergangsverwaltung keine Missachtung der Autonomie der Bürger dieses Staates. Im Gegenteil ist diese notwendig, um die Bedingungen zu schaffen, unter denen die Ausübung kollektiver Autonomie überhaupt erst möglich ist. 
Ein gewisses Unbehagen an Argumenten dieser Art erklärt sich daraus, dass sie historisch als eine der zentralen Rechtfertigungen des Kolonialismus missbraucht wurden. Wie Jennifer Pitts (2005) ausführlich gezeigt hat, waren gerade liberale Autoren geneigt, den Kolonialismus als notwendiges Instrument zur Zivilisierung der „Barbaren“ zu entschuldigen. Wie kleine Kinder, so etwa John Stuart Mill in seiner 1859 erstmals erschienenen Schrift „A Few Words on Non-Intervention“ (1984), seien die „Barbaren“ nicht in der Lage, ihr eigenes Schicksal in die Hände zu nehmen und insofern auf die wohlmeinende Anleitung durch die Kolonialisten angewiesen. Auch Lea Ypi betont in ihren jüngsten Ausführungen zum Thema „What's Wrong with Colonialism“ (2013), dass neben Gewalt und Ausbeutung das besondere moralische Übel des Kolonialismus darin bestanden habe, den vermeintlich „unzivilisierten Barbaren“ in politischer Hinsicht den Status als moralisch Gleiche zu verweigern. Mit Blick auf diese historische Dimension ist es darum wichtig zu betonen, dass das hier vorgeschlagene Argument für internationale Übergangsverwaltungen in keiner Weise die Behauptung impliziert, dass die betroffene Bevölkerung grundsätzlich nicht autonomiefähig sei. Das Argument ist lediglich, dass die besonderen Bedingungen eines extrem zerfallenen Staates es für die Bürger dieses Staates unmöglich machen, auf kollektiver Ebene Autonomie auszuüben (Applbaum 2007, S. 391).

Eine zweite Variante des Paternalismus-Einwandes bestreitet nicht, dass in besonders extremen Fällen von Staatszerfall eine internationale Übergangsverwaltung notwendig ist, warnt jedoch davor, diese Rechtfertigung auf allzu anspruchsvolle und dann doch wieder paternalistische Ziele auszuweiten. Im Kern findet sich dieses Argument bereits bei Rawls im „Recht der Völker“ (2002). Dort argumentiert er für eine Hilfspflicht gegenüber in Not geratenen Völkern, die er zugleich aber eng umgrenzt. Sobald ein Volk wieder über die notwendigen Institutionen verfüge, um die Menschenrechte seiner Bürger zu schützen - in Rawls Terminologie also zwar nicht liberal, aber doch „achtbar“ sei - sei weitere Hilfe nicht erforderlich. In der Tat, so Rawls weiter, verstoße der Versuch einer weiteren Einflussnahme mit dem Ziel der Entwicklung hin zu einer genuin liberalen Gesellschaft gegen das Recht auf kollektive Selbstbestimmung und sei insofern paternalistisch (Rawls 2002, S. 131-140).

In der Debatte um internationale Übergangsverwaltungen als eine besondere Form externer Hilfe hat sich Brian Orend in seinen Ausführungen zum ius post bellum eine sehr ähnliche Position zu eigen gemacht. ${ }^{9}$ Sein Vorschlag lautet dabei, dass aus der Theorie des „Gerechten Krieges“ hinreichend bekannte, jedoch letztlich rein formale, Prinzip der Proportionalität mit einer substanziellen Theorie der Menschenrechte zu kombinieren (Orend 2002, S. 56). Externe Einmischung beim Wiederaufbau eines im Zuge eines gerechten Krieges zerstörten Staates sei demnach in dem Maße zulässig, wie dies für einen zuverlässigen Menschenrechtsschutz notwendig sei (Orend 2002, S. 56). In ähnlicher Weise argumentiert Stefano Recchia (2009, 172-178), dass besonders umfassende Formen externer Einflussnahme wie etwa internationale Übergangsverwaltungen nur zum Schutz

9 Für einen Überblick zur Diskussion über das ius post bellum siehe Evans (2009) und Stahn (2008b). 
basaler Menschenrechte gerechtfertigt seien. Darüber hinaus, so Recchia, könne es zwar durchaus moralisch geboten sein, weitere Unterstützung zum Aufbau gerechter Institutionen zu leisten, derartige Unterstützung könne dann aber nur in Form der Kooperation mit lokalen Akteuren stattfinden. Recchia und Orend übernehmen von Rawls dessen vergleichsweise enges Verständnis von Menschenrechten, das diese auf grundlegende Freiheits- sowie basale Subsistenzrechte beschränkt (Rawls 2002, 96-99). ${ }^{10}$ Ein umfassenderes Verständnis von Menschenrechten, so Rawls und im Anschluss hieran Recchia und Orend, würde demzufolge eine paternalistische Verletzung des Rechts auf kollektive Selbstbestimmung bedeuten.

Diese Schlussfolgerung ist jedoch nicht zwingend. Versteht man, wie hier vorgeschlagen, Menschenrechte und das Recht auf kollektive Selbstbestimmung als komplementäre Instrumente zum Schutz menschlicher Grundinteressen, so ist es keinesfalls paternalistisch, wenn eine Übergangsverwaltung etwa auf dem Schutz grundlegender politischer Menschenrechte beharrt und notfalls von ihrer Autorität Gebrauch macht, um einen solchen Schutz zu gewährleisten. Wie oben dargestellt, ergibt sich der moralische Wert kollektiver Selbstbestimmung aus deren Bedeutung für die Autonomie der Mitglieder einer politischen Gemeinschaft. Beschränkt sich eine Übergangsverwaltung darauf, direkte Bedrohungen des menschlichen Grundinteresses an Autonomie zu verhindern, so ist eine solche Einschränkung des Rechts auf kollektive Selbstbestimmung daher nicht paternalistisch, sondern im Gegenteil moralisch geboten.

\subsection{Die Grenzen des Machbaren}

Ein ganz anderer Einwand lautet, dass die Idee internationaler Übergangsverwaltungen als Mittel zur Erfüllung der natürlichen Pflicht zur Gerechtigkeit zwar moralisch attraktiv, praktisch aber zum Scheitern verurteilt sei. Unmittelbar relevant für die moralische Diskussion von internationalen Übergangsverwaltungen erscheinen hier insbesondere zwei Ergebnisse der empirischen Forschung zum Statebuilding im Allgemeinen und zu internationalen Übergangsverwaltungen im Besonderen. ${ }^{11}$

Zum einen werden immer wieder die Schwierigkeiten betont, mit denen externe Akteure konfrontiert sind, wenn sie versuchen, politische, gesellschaftliche oder ökonomische Veränderungen in einer ihnen fremden Gesellschaft herbeizuführen. Ein zentrales Problem hierbei ist, dass es für externe Akteure oft schwierig ist, alle relevanten Informationen über die Besonderheiten der lokalen Umstände einzuholen (Stewart u. Knaus 2011, S. xix-xxvi). Dieses Problem verschärft sich noch angesichts der Komplexität der sozialen Prozesse in Gesellschaften, die tiefgreifenden Veränderungen ausgesetzt und von Gewalterfahrungen traumatisiert sind. In einer solchen Situation zu regieren, geschweige denn umfassende Reformen umzusetzen, ist selbst für lokale Akteure extrem schwierig. Für externe Ak-

10 Zu Rawls' Menschenrechtskonzeption siehe auch Reidy (2010).

11 Zur Bedeutung empirischer Forschung für (nicht-ideale) normative Theorie siehe unter anderem Farrelly (2007) und Simmons (2010). 
teure kann diese Aufgabe schnell unlösbar werden. Um unter diesen Bedingungen überhaupt noch handlungsfähig zu bleiben, greifen sie daher oft auf abstrakte, vermeintlich allgemeingültige Herangehensweisen zurück - häufig jedoch nur mit sehr beschränktem Erfolg (Bhuta 2008, S. 532).

Zum anderen weisen einige Autoren darauf hin, dass externe Unterstützungsmaßnahmen oft zu einer weiteren Schwächung lokaler Institutionen beitragen. Zumindest rhetorisch ist weithin unumstritten, dass das übergreifende Ziel externer Hilfsmaßnahmen der Aufbau und die Stärkung lokaler Strukturen sein sollte. In der Praxis zeigt sich jedoch, dass externe Akteure sich häufig mit einem tradeoff konfrontiert sehen: Während der Aufbau lokaler Strukturen langfristig als die nachhaltigere Lösung erscheint, ist kurzfristig die direkte Bereitstellung von Governance-Leistungen durch externe Akteure effektiver - und führt so nicht zuletzt auch zu schneller sichtbaren Ergebnissen. Die Gefahr ist dabei, dass sich auf diese Weise die lokale Bevölkerung an die externe Bereitstellung selbst grundlegender Regierungsleistungen gewöhnt, statt die eigene Regierung in die Verantwortung zu nehmen. Dies wiederum macht es der Regierung einfacher, sich ihrer Verantwortung zu entziehen und führt schließlich zu einer Situation, in der sich die Angewiesenheit auf externe Hilfe verstetigt (Barnett u. Zürcher 2009; Fukuyama 2004, S. 39-40).

Viele der Autoren, die sich empirisch mit den Effekten externer Hilfsleistungen beschäftigen, neigen zu Skepsis ob allzu ambitionierter Vorhaben. So schreibt etwa Marina Ottaway: „the international community is more likely to help postconflict societies stabilize and build a process that may eventually lead to democracy if it starts with modest but reachable goals than by insisting on a maximalist model likely to remain an unattainable vision" (2003, S. 321). Das Ziel des Aufbaus minimal gerechter Institutionen durch internationale Übergangsverwaltungen geht auf den ersten Blick über diese Forderung nach Mäßigung hinaus. Bei genauerem Hinsehen zeigt sich allerdings, dass das Ziel minimal gerechter Institutionen durchaus im Einklang mit den hier skizzierten Ergebnissen aus der empirischen Forschung steht. Zum einen beschränkt es die Verantwortung von Übergangsverwaltungen für die direkte Bereitstellung von Governance-Leistungen auf den Bereich des Schutzes basaler Sicherheitsrechte. Zwar ist auch diese Aufgabe anspruchsvoll, doch setzt sie andererseits nicht in gleichem Maße Vertrautheit mit lokalen Gegebenheiten voraus wie die Bereitstellung anspruchsvollerer Governance-Leistungen. Zum anderen reflektiert die eingeschränkte Rolle von Übergangsverwaltungen beim Aufbau minimal gerechter Institutionen die praktischen Grenzen externer Einflussnahme - und wirkt zugleich der Schwächung lokaler Institutionen entgegen. Die zentrale Rolle beim Wiederaufbau kommt hier schließlich lokalen Akteuren zu, die dafür auch gegenüber der lokalen Bevölkerung verantwortlich sind.

\section{Die Praxis internationaler Übergangsverwaltungen}

Im vorigen Abschnitt habe ich argumentiert, dass die Einrichtung einer internationalen Übergangsverwaltung aus der Perspektive der natürlichen Pflicht zur Gerechtigkeit dann moralisch gerechtfertigt und sogar geboten ist, wenn ein Staat 
nicht mehr in der Lage ist, jene Sicherheitsrechte seiner Bürger institutionell abzusichern, die in existenzieller Weise das menschliche Grundinteresse an körperlicher Unversehrtheit schützen: das Recht auf Leben und das Recht auf Schutz vor Folter. Weiter habe ich argumentiert, dass eine unter solchen Umständen eingerichtete internationale Übergangsverwaltung eine unmittelbare Verantwortung gegenüber der betroffenen Bevölkerung hat, eben diese Sicherheitsrechte zu schützen. Zudem ist es die Aufgabe einer internationalen Übergangsverwaltung, lokale Akteure beim Aufbau minimal gerechter Institutionen zu unterstützen. Dies beinhaltet auch die Verantwortung, sicherzustellen, dass diese Wiederaufbaumaßnahmen im Einklang mit dem Ziel minimal gerechter Institutionen stehen.

Will man diese Überlegungen auf die Praxis internationaler Übergangsverwaltungen übertragen, so wird schnell deutlich, dass dieser Vorgang keineswegs trivial ist. Der Zweck der hier formulierten moralischen Prinzipien besteht darin, einen systematischen Zugriff auf die moralischen Probleme der politischen Praxis zu ermöglichen. Dieser systematische Zugriff bedarf darüber hinaus jedoch der Ergänzung, Interpretation oder auch Korrektur durch die Art von empirisch gesättigtem Wissen, wie es vor allem die Sozialwissenschaften, aber auch etwa die Rechts- oder Geschichtswissenschaften, zur Verfügung stellen. Für diese Art der Zusammenführung moralischer Prinzipien und empirischer Einschätzungen braucht es schließlich jene Fähigkeit, die häufig mit dem wohl unvermeidbar unscharfen Begriff der „politischen Urteilskraft“ umschrieben wird.

Es ist nun an dieser Stelle nicht meine Absicht, in diesem Sinne eine umfassende Bewertung der Praxis internationaler Übergangsverwaltungen vorzunehmen. Anhand der internationalen Übergangsverwaltung im Kosovo möchte ich jedoch exemplarisch aufzeigen, wie die hier angestellten Überlegungen als Maßstab zur moralischen Bewertung solch konkreter Einzelfälle dienen können.

Der Fall des Kosovo zeigt dabei zunächst, dass es in der Tat Situationen gibt, in denen die Errichtung einer internationalen Übergangsverwaltung moralisch geboten ist. Die militärischen Auseinandersetzungen zwischen der NATO und Jugoslawien von März bis Juni 1999 hatten erhebliche Teile der Infrastruktur des Kosovos zerstört. Noch gravierender war jedoch der fast vollständige Zusammenbruch der öffentlichen Institutionen. Bis zum Ausbruch des Krieges waren diese unter dem Einfluss des jugoslawischen Zentralstaates fast ausschließlich mit Serben besetzt worden. Als die serbische Bevölkerung sich zum Ende des Krieges aus dem Süden des Kosovos zurückzog, brachen diese Strukturen daher zu großen Teilen in sich zusammen. So beschreibt Hansjörg Strohmeyer die Situation, die sich den ersten internationalen Beobachtern nach Ende der unmittelbaren Kriegshandlungen präsentierte, wie folgt: „It was apparent, within the first few days, that the previous law enforcement and judicial system had collapsed" (2001, S. 48). ${ }^{12}$ Schon bald kam es zu Racheakten von albanischen Kosovaren gegenüber den wenigen im Kosovo verbliebenen Serben. Einige Beobachter gehen davon aus, dass in den ersten Tagen und Wochen nach Ende der Kampfhandlungen mehr als

12 Siehe auch Amnesty International (2000, S. 264-267) und Human Rights Watch (2000, S. 316317). 
1.000 serbische Kosovaren ermordet wurden (Hehir 2006, S. 203-204). Hier zeigt sich die besondere moralische Dringlichkeit von Situationen extrem zerfallener Staatlichkeit, ebenso wie die Notwendigkeit, den daraus resultierenden Bedrohungen durch die Errichtung einer internationalen Übergangsverwaltung zu begegnen: In dieser Situation waren vor allem die Mitglieder der serbischen Minderheit, aber ebenso die albanischen Kosovaren gewaltsamen Übergriffen nahezu schutzlos ausgesetzt und befanden sich somit in existenzieller Gefahr. Zugleich verweist diese Situation auf die Verantwortung externer Akteure, vermittels einer internationalen Übergangsverwaltung ein extern gestütztes Gewaltmonopol zu errichten, um so die Bevölkerung des Kosovos vor gewaltsamen Übergriffen zu schützen.

Mit besonderer Deutlichkeit zeigt sich am Fall des Kosovo aber auch, dass sich die Frage nach der moralischen Notwendigkeit einer internationalen Übergangsverwaltung zunächst unabhängig davon stellt, wie man die Umstände bewertet, die eine solche Übergangsverwaltung notwendig erscheinen lassen. So ist es durchaus möglich, die militärische Intervention der NATO zu kritisieren und dennoch zu dem Schluss zu kommen, dass die Situation im Juni 1999 die Errichtung einer internationalen Übergangsverwaltung erforderlich gemacht hat. Mehr noch: Die Entscheidung, in einer solchen Situation eine internationale Übergangsverwaltung zu errichten, impliziert keinesfalls im Umkehrschluss eine rückwirkende Rechtfertigung der militärischen Intervention der NATO. Ohne die strategischen Interessen der beteiligten Akteure auszublenden, erscheint es durchaus plausibel, die Entscheidungen des UN-Sicherheitsrates bezüglich des Kosovo auf eben diese Weise zu deuten: Obwohl der Sicherheitsrat sich zunächst geweigert hatte, die NATO-Intervention zu mandatieren, autorisierte er im Lichte der neu entstandenen Situationen nach Ende der Kampfhandlungen die United Nations Interim Administration Mission in Kosovo (UNMIK). ${ }^{13}$

Das in UN-Resolution 1244 formulierte Mandat von UNMIK sah dabei zum einen vor, dass UNMIK abgesichert durch die Militäreinheiten der Kosovo Force (KFOR) für eine Übergangsphase die Verwaltung des Kosovo übernehmen sollte. Zum anderen sah das Mandat aber auch den Aufbau rechtsstaatlicher und demokratischer Strukturen vor, die eine politische Lösung des in UN-Resolution 1244 bewusst ausgeklammerten Konfliktes um die Unabhängigkeit des Kosovo von Jugoslawien ermöglichen sollte. Diese Aufgabenbeschreibung steht durchaus im Einklang mit den hier diskutierten moralischen Prinzipien zu den Zielen internationaler Übergangsverwaltungen. Allerdings zeigte sich bald, dass die Praxis von UNMIK diesen selbst gesetzten Zielen nur eingeschränkt entsprach.

Erstens war UNMIK in einigen Situationen nicht in der Lage, die Sicherheit der lokalen Bevölkerung zu gewährleisten. Nach der Einrichtung der internationalen Übergangsverwaltung hatte sich die Sicherheitslage zunächst deutlich gebessert. 2004 jedoch kam es zu massiven und gewaltsamen Unruhen, die sich gegen die serbische Minderheit richteten. Die im Kosovo stationierten Militär- und Polizeieinheiten beschränkten sich in dieser Situation mit wenigen Ausnahmen auf die sogenannte „force protection“, also den Schutz der eigenen Einheiten. Erst nach

13 Siehe UN Sicherheitsratsresolution 1244, 10. Juni 1999, S/RES/1244. 
Tagen, und nachdem 19 Menschen getötet worden waren, unternahm die Übergangsverwaltung mit Unterstützung von weiteren Einheiten aus Bosnien-Herzegowina die notwendigen Maßnahmen, um die Ausschreitungen zu beenden (King u. Mason 2006, S. 5-20). Dieses Versagen von UNMIK wiegt dabei moralisch besonders schwer. Wie zuvor argumentiert, ist eine internationale Übergangsverwaltung als Form von Fremdherrschaft nur dann moralisch rechtfertigbar, wenn dies das einzige Mittel ist, um die Sicherheitsrechte der Bürger eines zerfallenen Staates zu schützen. Zeigt sich eine internationale Übergangsverwaltung selbst als unfähig, diese Rechte zu schützen, stellt dies mithin sehr grundsätzlich ihre moralische Rechtfertigung in Frage.

Zweitens kam UNMIK in einigen wichtigen Hinsichten nicht der Verantwortung einer internationalen Übergangsverwaltung für den Wiederaufbau minimal gerechter Institutionen nach. Ohne Zweifel wurde diese Aufgabe durch die ungeklärte Status-Frage weiter erschwert, war UNMIK doch durch Resolution 1244 darauf festgelegt, jegliche Vorfestlegung in dieser Frage zu unterbinden. Das unter der Leitung von UNMIK entwickelte „Constitutional Framework for Provisional Self-Government in Kosovo" macht am Beispiel des Verfassungsrechts schon sprachlich auf diese Komplikation aufmerksam. ${ }^{14}$ Dies entschuldigt jedoch nicht die unzureichenden Bemühungen von UNMIK, die weitverbreitete ethnische Diskriminierung in den Sicherheitsinstitutionen des Kosovo im Sinne des Aufbaus minimal gerechter Institutionen zu überwinden. Nach wiederholten Berichten über die Diskriminierung von Mitgliedern der serbischen Minderheit durch das von Kosovo-Albanern dominierte Justizsystem bemühte sich UNMIK zwar, diesem Problem durch die Stärkung der Rolle internationaler Richter zu stärken. Diese Maßnahmen waren jedoch nach Ansicht vieler Beobachter zu beschränkt, um eine tatsächliche Veränderung bewirken zu können (Friedrich 2005, S. 263264; Hartmann 2003). Ähnliche Probleme zeigten sich beim Wiederaufbau des Polizeisystems. Auch hier konnte die strukturelle Diskriminierung der serbischen Minderheit nicht verhindert werden. Dies zeigte sich mit besonderer Deutlichkeit, als im Laufe der bereits erwähnten Ausschreitungen die kosovarische Polizei in den meisten Fällen keinerlei Anstalten unternahm, die Mitglieder der serbischen Minderheit zu beschützen.

Drittens verletzte UNMIK zugleich in einigen Hinsichten das Recht der Kosovaren auf kollektive Selbstbestimmung, indem die Übergangsverwaltung sich in unzulässiger Weise in den lokalen Wiederaufbauprozess einmischte. Ein Beispiel hierfür ist die von UNMIK durchgesetzte Einführung eines Verhältniswahlrechts wider den erklärten Willen der lokalen Parteien, die sich ganz überwiegend für ein Mehrheitswahlrecht ausgesprochen hatten (Tansey 2007, S. 138). Die wohl dahinterstehende Absicht, die Rolle politischer Minderheiten im politischen Prozess zu stärken, ist zwar nachvollziehbar, rechtfertigt aber nicht eine solch extreme Form des „electoral engineering “. ${ }^{15}$ Ein weiteres Beispiel sind die Aktivitäten von

14 Siehe UNMIK Regulation 2001/9: On a Constitutional Framework for Provisional Self-Government in Kosovo.

15 Zur Diskussion über „electoral engineering“ siehe auch Belloni (2004), Manning u. Antic (2003) und Reilly (2001). 
UNMIK zur Privatisierung jener staatseigenen Betriebe, die zu Zeiten Jugoslawiens den größten Teil der Wirtschaft im Kosovo ausmachten. Obwohl es sogar unter den Mitarbeitern von UNMIK zu Kontroversen über die rechtliche Grundlage dieser Privatisierungsmaßnahmen kam, setzte UNMIK letztlich die Privatisierung der meisten Betriebe durch. Ob dies wirtschaftspolitisch notwendig war, sei dahingestellt, erkennbar verletzt eine solch irreversible Entscheidung über die zukünftige Wirtschaftsordnung durch eine internationale Übergangsverwaltung jedoch das Recht der betroffenen Gesellschaft auf kollektive Selbstbestimmung.

Die moralische Bilanz der internationalen Übergangsverwaltung im Kosovo ist so insgesamt durchwachsen. Obwohl die Einrichtung einer internationalen Übergangsverwaltung nach dem Ende des Krieges 1999 gerechtfertigt erscheint, verletzte UNMIK in der Folge auf unterschiedliche Weise und oft mit dramatischen Folgen für die Betroffenen die moralischen Anforderungen an internationale Übergangsverwaltungen. Ohne dies hier in der gebotenen Ausführlichkeit darstellen zu können, scheint mir, dass der Fall Kosovo in dieser Hinsicht repräsentativ für die bisherige Praxis internationaler Übergangsverwaltungen ist. Die hier formulierten moralischen Anforderungen lassen sich insofern als Kritik an der bisherigen Praxis internationaler Übergangsverwaltungen verstehen. Da jedoch davon auszugehen ist, dass es auch in Zukunft Situationen von extremem Staatszerfall geben wird, sind die hier angestellten Überlegungen jedoch zugleich als Appell zu verstehen, neue Wege für die Praxis internationaler Übergangsverwaltungen zu suchen. In diesem Sinne gilt für internationale Übergangsverwaltungen, was Roland Paris mit Blick auf extern geleitete friedensbildende Maßnahmen im Allgemeinen bemerkt: „[P]eacebuilding is tremendously complex and prone to unanticipated consequences, yet it is also too important to lose or abandon" (2010, S. 365).

\section{Schlussüberlegungen}

Kann eine internationale Übergangsverwaltung eine Form der Regierung für das Volk sein, obwohl sie doch schon laut Definition keine Regierung durch das Volk geschweige denn des Volkes ist? Und kann eine solche Regierung für das Volk den in dieser Formulierung schon sprachlich fast unausweichlich scheinenden Paternalismus vermeiden, der letztlich eben jene liberalen Werte verletzen würde, die doch zugleich die moralische Rechtfertigung für diese besondere Form der Fremdherrschaft liefern sollen? In diesem Artikel habe ich mich bemüht zu zeigen, dass die Idee einer natürlichen Pflicht zur Gerechtigkeit uns in der Tat Gründe dafür liefert, in Situationen von extremem Staatszerfall eine internationale Übergangsverwaltung einzurichten, um so die Bürger des betroffenen Staates vor existenziellen Gefahren zu bewahren. Zugleich verweist die natürliche Pflicht zur Gerechtigkeit aber auch auf die Grenzen, die einzuhalten sind, um paternalistische Bevormundung zu vermeiden. So müssen sich internationale Übergangsverwaltungen in ihren Zielen darauf beschränken, die Sicherheit der lokalen Bevölkerung zu gewährleisten und den von lokalen Akteuren durchzuführenden Wiederaufbau minimal gerechter Institutionen abzusichern. Wie im vorigen Abschnitt ausgeführt, mangelt es in der Praxis jedoch häufig an eben dieser moralisch gebotenen Selbstbeschränkung. Allzu oft scheint die Versuchung letztlich doch zu 
groß, von der unheimlichen Machtfülle Gebrauch zu machen, die sich externen Akteuren in diesen Situationen bietet.

Eine gängige Forderung lautet daher, auch im Falle von internationalen Übergangsverwaltungen die Rolle multilateraler Institutionen wie der Vereinten Nationen zu stärken, um so die Macht einzelner Staaten zu beschränken. ${ }^{16}$ Zurecht erweitert sich damit der Gegenstand der Debatte: Mein Anliegen in diesem Artikel war es, zu klären, unter welchen Bedingungen internationale Übergangsverwaltungen ein Mittel sein können, um die Anforderungen der natürlichen Pflicht zur Gerechtigkeit zu erfüllen. Dies wirft in einem nächsten Schritt die Frage auf, welchen Akteuren oder Institutionen konkret die Aufgabe zugeschrieben werden sollte, diese Anforderungen zu erfüllen. Diese Frage stellt sich dabei nicht nur für den besonderen Fall von internationalen Übergangsverwaltungen, sondern letztlich für all jene Verpflichtungen, die heute mit dem Begriff der „responsibility to protect" in Verbindung gebracht werden (Pattison 2010).

Das oben vorgeschlagene Modell moralischer Arbeitsteilung gibt hierfür erste Anhaltspunkte, indem es den Umgang mit zerfallenden Staaten zunächst grundsätzlich zu einer Angelegenheit der Staatengemeinschaft in Gänze erklärt. Um auf dieser Grundlage einzelnen Staaten spezifischere Verpflichtungen zuzuweisen, bedarf es jedoch erkennbar weiterer Erwägungen: So verfügen erstens nicht alle Staaten in gleicher Weise über die notwendigen Kenntnisse und Ressourcen, um entsprechende Hilfsleistungen zu erbringen. Zweitens gilt es, den historischen Kontext der Beziehungen zwischen den betreffenden Staaten zu betrachten. Historisches Unrecht etwa kann einem Staat besondere Verpflichtungen auferlegen, und ihn zugleich als moralisch und praktisch ungeeignet für bestimmte Formen der externen Hilfsleistung erscheinen lassen. Drittens liegt es schließlich nahe, auch Fragen der Verteilungsgerechtigkeit mit in den Blick zu nehmen, also die Kosten für entsprechende Hilfsleistungen fair zwischen den Staaten zu verteilen. Viertens ist, wie bereits angesprochen, zu klären, inwiefern Staaten ihrer Verantwortung direkt oder vermittels der Unterstützung von gemeinsamen Institutionen der Staatengemeinschaft gerecht werden sollten.

Zusammengenommen bieten diese Erwägungen eine Reihe von Anhaltspunkten dafür, wie sich die Idee einer globalen moralischen Arbeitsteilung genauer bestimmen lässt. Für die weitere Debatte über die globalen Implikationen der Idee einer natürlichen Pflicht zur Gerechtigkeit erscheint es somit als zentrale Herausforderung, diese Erwägungen zur Allokation spezifischer Verpflichtungen und die ihnen zugrundeliegenden Prinzipien genauer zu erfassen und miteinander in Beziehung zu setzen.

16 Vgl. hierzu etwa Walzers (2004) Anmerkungen zu der Situation im Irak nach dem Krieg 2003. Wolfgang Seibel (2008) hingegen betont gerade mit Blick auf internationale Übergangsverwaltungen, dass multilaterale Institutionen ebenfalls häufig im Sinne der strategischen Interessen mächtiger Staaten missbraucht werden. 


\section{Literatur}

Amnesty International. 2000. Amnesty International Report 2000. The State of the World's Human Rights. London: Amnesty International Publications.

Anderson, Elizabeth S. 1999. What Is the Point of Equality? Ethics 109: 287-337.

Anderson, Joel, und John Christman. 2005. Introduction. In dies.: Autonomy and the Challenges to Liberalism. New Essays, 1-23. Cambridge: Cambridge University Press.

Applbaum, Arthur Isak. 2007. Forcing A People to Be Free. Philosophy \& Public Affairs 35: 359-400.

Arneson, Richard J. 1980. Mill versus Paternalism. Ethics 90: 470-489.

Bader, William B. 1966. Austria between East and West, 1945-1955. Stanford, CA: Stanford University Press.

Bain, William. 2003. Between Anarchy and Society: Trusteeship and the Obligations of Power. Oxford: Oxford University Press.

Barnett, Michael, und Christoph Zürcher. 2009. The Peacebuilder's Contract. How External Statebuilding Reinforces Weak Statehood. In The Dilemmas of Statebuilding: Confronting the Contradictions of Postwar Peace Operations. Hrsg. Roland Paris und Timothy Sisk, 23-52. London: Routledge.

Beitz, Charles. 2009. The Idea of Human Rights. Oxford: Oxford University Press.

Bellamy, Alex J. 2010. The Responsibility to Protect - Five Years On. Ethics \& International Affairs 24: 143-169.

Belloni, Roberto. 2004. Peacebuilding and Consociational Electoral Engineering in Bosnia and Herzegovina. International Peacekeeping 11: 334-353.

Bhuta, Nehal. 2008. Against State-Building. Constellations 15: 517-542.

Boothby, Derek. 2004. The Political Challenges of Administering Eastern Slavonia. Global Governance 10: 37-51.

Börzel, Tanja, und Thomas Risse. 2010. Governance Without a State: Can it work? Regulation \& Governance 4: 113-134.

Bose, Sumantra. 2002. Bosnia after Dayton. Nationalist Partition and International Intervention. Oxford: Oxford University Press.

Bothe, Michael, und Thilo Marauhn. 2002. UN Administration of Kosovo and East Timor: Concept, Legality and Limitations of Security Council-Mandated Trusteeship Administration. In Kosovo and the International Community: A Legal Assessment, Hrsg. Christian Tomuschat, 217-242. Den Haag: Kluwer Law International.

Buchanan, Allen. 2004. Justice, Legitimacy, and Self-Determination. Moral Foundations for International Law. Oxford: Oxford University Press.

Call, Charles. 2010. Beyond the ,Failed State': Toward Conceptual Alternatives. European Journal of International Relations 17: 303-326.

Caplan, Richard. 2004a. International Authority and State Building: The Case of Bosnia and Herzegovina. Global Governance 10: 53-65.

Caplan, Richard. 2004b. Partner or Patron? International Civil Administration and Local Capacity-Building. International Peacekeeping 11: 229-247.

Caplan, Richard. 2005. International Governance of War-Torn Territories. Rule and Reconstruction. Oxford: Oxford University Press.

Carafano, James Jay. 2002. Waltzing into the Cold War. The Struggle for Occupied Austria. College Station, TX: Texas A\&M University Press. 
Chandler, David. 2002. From Kosovo to Kabul. Human Rights and International Intervention. London: Pluto Press.

Chesterman, Simon. 2002. East Timor in Transition: Self-Determination, State-Building and the United Nations. International Peacekeeping 9: 45-76.

Chesterman, Simon. 2004 You, the People. The United Nations, Transitional Administration, and State-Building. Oxford: Oxford University Press.

Christman, John. 2009. The Politics of Persons. Individual Autonomy and Socio-historical Selves. Cambridge: Cambridge University Press.

Dawisha, Adeed. 2009. Iraq. A Political History From Independence to Occupation. Princeton, NJ: Princeton University Press.

Diefendorf, Jeffry M., Axel Frohn, und Hermann-Josef Rupierer (Hrsg.). 1993. American Policy and the Reconstruction of West Germany, 1945-1955. Cambridge: Cambridge University Press.

Dower, John W. 1999. Embracing Defeat. Japan in the Wake of World War II. New York, NY: W.W. Norton \& Co.

Dworkin, Gerald. 1983. Paternalism. In Paternalism, Hrsg. Rolf Sartorius, 19-34. Minneapolis, MN: University of Minnesota Press.

Dworkin, Gerald. 1989. The Concept of Autonomy. In The Inner Citadel, Hrsg. John Christman, 54-62. Oxford: Oxford University Press.

Evans, Mark. 2009. Moral Responsibilites and the Conflicting Demands of Jus Post Bellum. Ethics \& International Affairs 23: 147-164.

Farrelly, Colin. 2007. Justice in Ideal Theory: A Refutation. Political Studies 55: 844-864.

Feinberg, Joel. 1984. The Moral Limits of the Criminal Law. Vol. 1: Harm to Others. Oxford: Oxford University Press.

Feinberg, Joel. 1986. The Moral Limits of the Criminal Law. Vol. 3: Harm to Self. Oxford: Oxford University Press.

Fox, Gregory H. 2008. Humanitarian Occupation. Cambridge: Cambridge University Press.

Frankfurt, Harry G. 1971. Freedom of the Will and the Concept of a Person. The Journal of Philosophy 68: 5-20.

Friedrich, Jürgen. 2005. UNMIK in Kosovo: Struggling with Uncertainty. Max Planck Yearbook of United Nations Law 9: 225-293.

Fukuyama, Francis. 2004. State-Building. Governance and World Order in the 21st Century. Ithaca, NY: Cornell University Press.

Gheciu, Alexandra, und Jennifer Welsh. 2009. Postwar Justice and the Responsibility to Rebuild. Ethics \& International Affairs 23: 115-120.

Goodin, Robert E. 1988. What is So Special about Our Fellow Countrymen? Ethics 98: 663-686.

Grant, Ruth. 2012. Strings Attached. Untangling the Ethics of Incentives. Princeton, NJ: Princeton University Press.

Grill, Kalle. 2007. The Normative Core of Paternalism. Res Publica 13: 441-458.

Gruss, Daniel. 2005. UNTEA and West New Guinea. Max Planck Yearbook of United Nations Law 9: 97-126.

Hartmann, Michael E. 2003. International Judges and Prosecutors in Kosovo (United States Institute of Peace Special Report No 112). Washington, DC: United States Institute of Peace. 
Hehir, Aidan. 2006. Autonomous Province Building. Identification Theory and the Failure of UNMIK. International Peacekeeping 13: 200-213.

Höffe, Otfried. 1998. Transzendentaler Tausch - eine Legitimationsfigur für die Menschenrechte? In Philosophie der Menschenrechte, Hrsg. Stefan Gosepath und Georg Lohmann, 29-47. Frankfurt a. M.: Suhrkamp Verlag.

Holzgrefe, J.L., und Robert O. Keohane (Hrsg.). 2003. Humanitarian Intervention: Ethical, Legal and Political Dilemmas. Cambridge: Cambridge University Press.

Human Rights Watch. 2000. World Report 2000. New York, NY: Human Rights Watch.

Jackson, Robert. 2004. International Engagement in War-Torn Countries. Global Governance 10: 21-36.

Jarausch, Konrad H. 2006. After Hitler. Recivilizing Germans, 1945-1995. Oxford: Oxford University Press.

Kawai, Kazuo. 1979. Japan's American Interlude. Chicago, IL: University of Chicago Press.

King, Iain, und Whit Mason. 2006. Peace at Any Price. How the World Failed Kosovo. Ithaca, NY: Cornell University Press.

Ladwig, Bernd. 2010. Menschenwürde als Grund der Menschenrechte? Eine Kritik an Kant und über Kant hinaus. Zeitschrift für politische Theorie 1: 51-69.

Mackenzie, Catriona, und Natalie Stoljar. 2000. Introduction. Autonomy Refigured. In dies.: Relational Autonomy. Feminist Perspectives on Autonomy, Agency, and the Social Self, 3-31. Oxford: Oxford University Press.

Manning, Carrie, und Miljenko Antic. 2003. Lessons from Bosnia and Herzegovina: The Limits of Electoral Engineering. Journal of Democracy 14: 45-59.

Marneffe, Peter de. 2006. Avoiding Paternalism. Philosophy \& Public Affairs 34: 68-94.

Mayall, James. 2011. The European Empires and International Order. Model or Trap? In The New Protectorates. International Tutelage and the Making of Liberal States, Hrsg. James Mayall und Ricardo Soares de Oliveria, 49-65. London: C. Hurst \& Co.

Mill, John Stuart. [1859] 1984. A Few Words on Non-Intervention. In The Collected Works of John Stuart Mill. Volume XXI - Essays on Equality, Law, and Education, Hrsg. John M. Robson, 111-124. Toronto: University of Toronto Press.

Miller, David. 2007. National Responsibility and Global Justice. Oxford: Oxford University Press.

Neuhäuser, Christian. 2011. Humiliation. The Collective Dimension. In Humiliation, Degradation, Dehumanization, Hrsg. Paulus Kaufmann, Hannes Kuch und Christian Neuhäuser, 21-36. Dordrecht: Springer.

Nickel, James. 2007. Making Sense of Human Rights. Malden, MA: Blackwell.

Nussbaum, Martha C. 2000. Women and Human Development. The Capabilities Approach. Cambridge: Cambridge University Press.

Nussbaum, Martha C. 2011. Perfectionist Liberalism and Political Liberalism. Philosophy \& Public Affairs 39: 3-45.

Orend, Brian. 2002. Justice After War. Ethics \& International Affairs 16: 43-56.

Ottaway, Marina. 2003. Promoting Democracy after Conflict: The Difficult Choices. International Studies Perspectives 4: 312-322.

Patrick, Stewart. 2006. Weak States and Global Threats: Fact or Fiction? The Washington Quarterly 29: 27-53.

Paris, Roland. 2010. Saving Liberal Peacebuilding. Review of International Studies 36: 337-365. 
Pattison, James. 2010: Humanitarian Intervention and the Responsibility To Protect: Who Should Intervene? Oxford: Oxford University Press.

Pettit, Philip, und David Schweikard. 2006. Joint Actions and Group Agents. Philosophy of the Social Sciences 36: 18-39.

Pitts, Jennifer. 2005. A Turn to Empire. The Rise of Imperial Liberalism in Britain and France. Princeton, NJ: Princeton University Press.

Pogge, Thomas. 2004. ,Assisting' the Global Poor. In The Ethics of Assistance, Hrsg. Deen K. Chatterjee, 260-288. Cambridge: Cambridge University Press.

Pogge, Thomas. 2005. Severe Poverty as a Violation of Human Rights. Ethics \& International Affairs 19: 55-83.

Rawls, John. 1975. Eine Theorie der Gerechtigkeit. Frankfurt a. M.: Suhrkamp Verlag.

Rawls, John. 2002. Das Recht der Völker. Berlin: de Gruyter.

Raz, Joseph. 1986. The Morality of Freedom. Oxford: Oxford University Press.

Recchia, Stefano. 2009. Just and Unjust Postwar Reconstruction: How Much External Interference Can Be Justified. Ethics \& International Affairs 23: 165-187.

Reidy, David A. 2010. Human Rights and Liberal Toleration. Canadian Journal of Law and Jurisprudence 23: 287-317.

Reilly, Benjamin. 2001. Democracy in Divided Societies. Electoral Engineering for Conflict Management. Cambridge: Cambridge University Press.

Rotberg, Robert I. (Hrsg.). 2004. When States Fail. Causes and Consequences. Princeton, NJ: Princeton University Press.

Saltford, John. 2003. The United Nations and the Indonesian Takeover of West Papua, 1962-1969. The Anatomy of Betrayal. London: Routledge.

Sassòli, Marco. 2005. Legislation and Maintenance of Public Order and Civil Life by Occupying Powers. European Journal of International Law 16: 661-694.

Seibel, Wolfgang. 2008 Moderne Protektorate als Ersatzstaat: UN-Friedensoperationen und Dilemmata internationaler Übergangsverwaltungen. In Governance in einer sich wandelnden Welt. PVS Sonderheft 41, Hrsg. Gunnar Folke Schuppert und Michael Zürn, 499-530. Baden-Baden: Nomos.

Shue, Henry. 1980. Basic Rights. Subsistance, Affluence, and U.S. Foreign Policy. Princeton, NJ: Princeton University Press.

Shue, Henry. 1988. Mediating Duties. Ethics 98: 687-704.

Simmons, A. John. 2010. Ideal and Nonideal Theory. Philosophy \& Public Affairs 38: 5-36.

Stahn, Carsten. 2008a. The Law and Practice of International Territorial Administration. Versailles to Iraq and Beyond. Cambridge: Cambridge University Press.

Stahn, Carsten. 2008b. Jus Post Bellum: Mapping the Discipline(s). American University International Law Review 23: 311-347.

Stewart, Rory, und Gerald Knaus. 2011. Can Intervention Work? New York, NY: W.W. Norton \& Co.

Stilz, Anna. 2009. Liberal Loyalty. Freedom, Obligation, and the State. Princeton, NJ: Princeton University Press.

Strohmeyer, Hansjörg. 2001. Collapse and Reconstruction of a Judicial System. The United Nations Mission in Kosovo and East Timor. The American Journal of International Law 95: 46-63. 
Suhrke, Astri. 2009. The Dangers of a Tight Embrace. Externally Assisted Statebuilding in Afghanistan. In The Dilemmas of Statebuilding: Confronting the Contradictions of Postwar Peace Operations, Hrsg. Roland Paris und Timothy D. Sisk, 227-251. London: Routledge.

Tansey, Oisín. 2007. Democratization without a State: Democratic Regime-building in Kosovo. Democratization 14: 129-150.

Waldron, Jeremy. 1993. Special Ties and Natural Duties. Philosophy \& Public Affairs 22: 3-30.

Walzer, Michael. 2004. Just and Unjust Occupations. Dissent 51: 61-63.

Wambaugh, Sarah. 1940. The Saar Plebiscite, with a Collection of Official Documents. Harvard University Press: Cambridge University Press.

Wellman, Christopher H. 1996 Liberalism, Samaritanism, and Political Legitimacy. Philosophy \& Public Affairs 25: 211-237.

Weller, Marc. 2008. Kosovo's Final Status. International Affairs 84: 1223-1243.

Wheeler, Nicholas, und Tim Dunne. 2001. East Timor and the New Interventionism. International Affairs 77: 805-827.

Wilde, Ralph. 2001. From Danzig to East Timor and Beyond: The Role of International Territorial Administration. The American Journal of International Law 95: 583-606.

Wolfrum, Rüdiger. 2005. Iraq - from Belligerent Occupation to Iraqi Exercise of Sovereignty: Foreign Power versus International Community Interference. Max Planck Yearbook of United Nations Law 9: 1-45.

Ypi, Lea. 2013. What's Wrong with Colonialism. Philosophy \& Public Affairs 41: 158191.

\section{Autorenangaben}

Daniel Jacob

Freie Universität Berlin, Sonderforschungsbereich 700,

Binger Str. 40,

14197 Berlin,

daniel.jacob@fu-berlin.de 